\title{
Videos in der Zeitschrift für Orthopädie und Unfallchirurgie - eine neue Rubrik
}

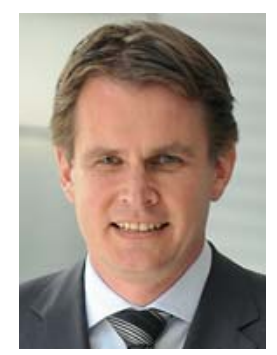

U. Stöckle

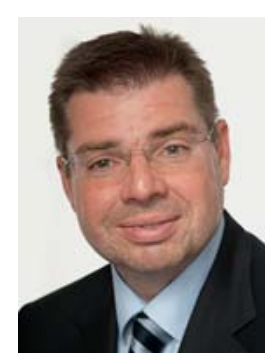

D. C. Wirtz

\section{Bibliografie}

DOI http://dx.doi.org/ 10.1055/s-0033-1350910 Z Orthop Unfall 2013; 151: 452453 @ Georg Thieme Verlag KG Stuttgart · New York . ISSN 1864-6697

\section{Korrespondenzadressen Univ.-Prof. Dr. Ulrich Stöckle Ärztlicher Direktor BGU Klinik Tübingen Schnarrenbergstraße 96 72076 Tübingen Tel.: 0 7071/606-1001 Fax: 07071/606-1002 ustoeckle@bgu-tuebingen.de}

\section{Univ.-Prof. Dr. med.} Dieter C. Wirtz

Direktor der Klinik und Poliklinik für Orthopädie und Unfallchirurgie Universitätsklinikum Bonn Sigmund-Freud-Straße 25 53105 Bonn

Tel.: 0228/287-14170

Fax: 0228/287-14175

dieter.wirtz@ukb.uni-bonn.de
Die Zeitschrift für Orthopädie und Unfallchirurgie versteht sich als wissenschaftliche Publikationsplattform in Orthopädie und Unfallchirurgie, die das gesamte inhaltliche Spektrum in unserem gemeinsamen Fachgebiet abdeckt.

Die Zeitschrift für Orthopädie und Unfallchirurgie greift neben den wissenschaftlichen Entwicklungen auch regelmäßig das Thema der Fort- und Weiterbildung auf, nicht zuletzt durch die Rubrik
„Junges Forum“, das sich als Sprachrohr unserer Weiterbildungsassistenten versteht. So finden Sie auch in dieser Ausgabe einen Beitrag, der sich diesem Thema widmet.

Um das Thema Weiterbildung auch künftig zu begleiten, freuen wir uns, dass wir Ihnen ab der aktuellen Ausgabe 5/2013 eine neue Rubrik in unserer Zeitschrift vorstellen können:

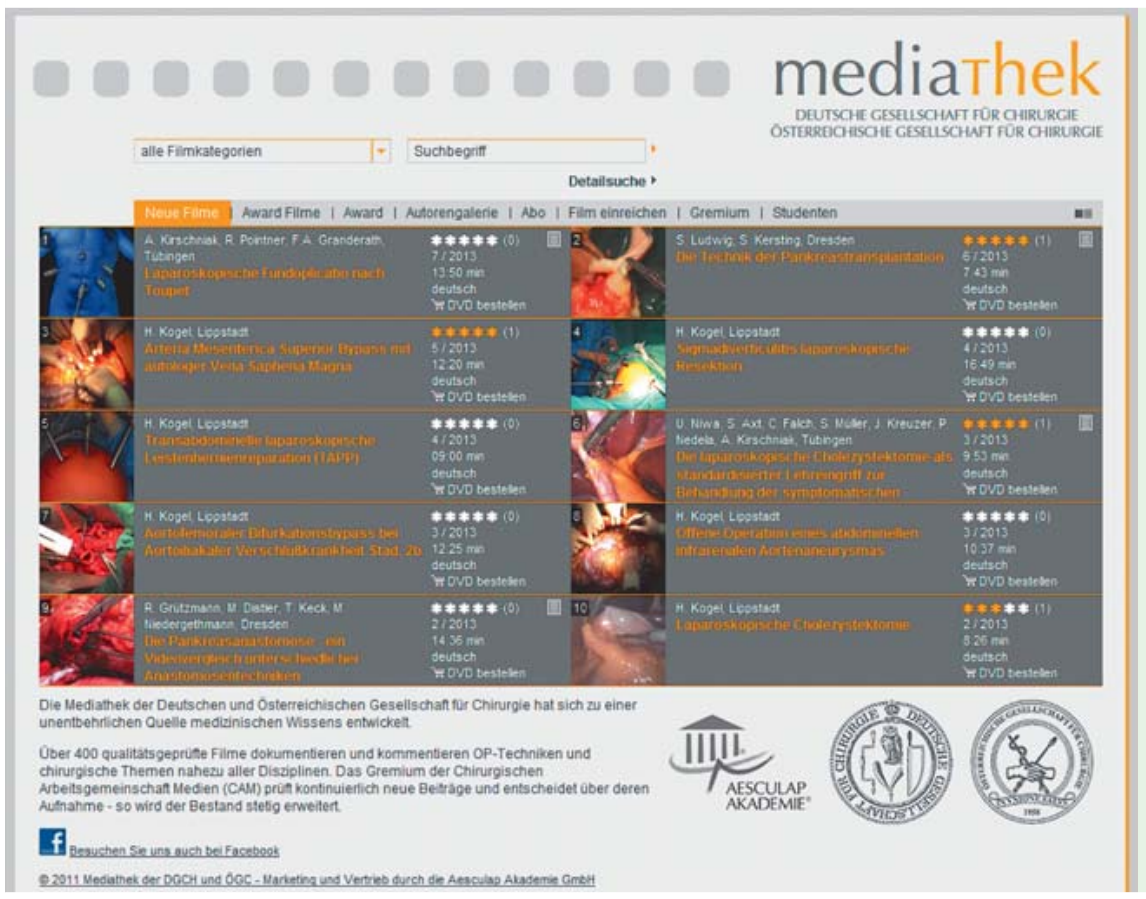

Abb. 1

Mediathek der DGCH.

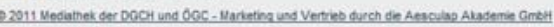

Intramedulläre Stabilisierung von Klavikulafrakturen

Intramedullary Stabilisation of Clavicula Fractures

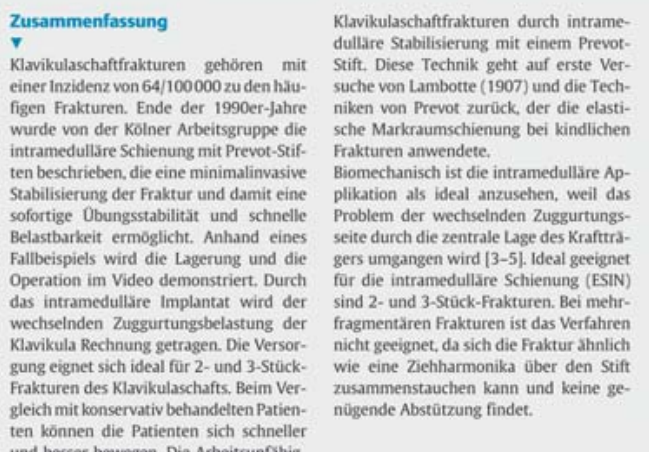

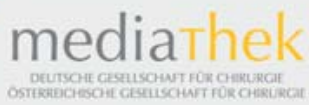

Anăsthesie befindet sich am FuBende und beatmet den Patienten mit langen Schläuchen, sodass der Operateur und e steht, gut beweglich sind. Fine Untertorerung des Schlisselbeins mit einerdurchleuchtbaren Kissen kann die Reposition erleichtern.

Vor der Operation wird das Jugulum und das Durch Verschieben der Haut gelinger den Schnitt, der jetzt am mediolen En den Schinitt, der jetzt anmer medalen Ende veset? wird, nicht otber dem spiteren Stiftende zul phatzieren Der Sctnitt ist nur wenige Millimeter lang Die darunter befindliche Klwikula wind mie daruntes mm-Bohrer unikortikat eroffoet und der mm-Bohrer unikortikal eröfinet und der ovales loch au erneugen Kleine Pfriene
Abb. 2

Der Link am Ende des Videopapers führt zum Video in der Mediathek. 
Im Rahmen einer gemeinsamen Kooperation der Mediathek der DGCH und der ÖGC, die maßgeblich von der Aesculapakademie unterstützt wird, und der Zeitschrift für Orthopädie und Unfallchirurgie werden künftig Videopapers in der Zeitschrift publiziert, die sich auf ein Video in der Mediathek ( $\triangle$ Abb. 1 und 2) beziehen. Bei der Initiierung der neuen Rubrik wurden wir maßgeblich von Herrn PD Dr. Stephan Kersting, Vorsitzender der Chirurgischen Arbeitsgemeinschaft Medien (CAM), und Herrn Professor Dr. Axel Prokop, der innerhalb der CAM für das Fachgebiet Orthopädie und Unfallchirurgie verantwortlich zeichnet, unterstützt. Herr Professor Dr. Axel Prokop wird künftig gemeinsam mit Herr Dr. Gereon Schiffer die neue „Videorubrik“ als Herausgeber begleiten. Wir begrüßen sie herzlich im Herausgeberboard der Zeitschrift für Orthopädie und Unfallchirurgie und freuen uns auf eine gute und erfolgreiche Zusammenarbeit.
Als Leser der Zeitschrift für Orthopädie und Unfallchirurgie können Sie künftig direkt aus der Online-Version des Videoartikels auf das dazugehörige Video in der Vollversion innerhalb der Mediathek zugreifen. Profitieren Sie von dieser neuen Möglichkeit zur Fort- und Weiterbildung. Als Autor und Produzent eines Videobeitrags haben Sie auf diesem Weg die Möglichkeit, nicht nur Ihr Video bei der Mediathek einzureichen, sondern dieses gleichzeitig zur Basis einer Publikation der Zeitschrift für Orthopädie und Unfallchirurgie zu machen, die damit in allen relevanten Datenbanken indexiert ist und recherchierbar wird. Wir freuen uns auf Ihre Beiträge und hoffen, dass Sie diese attraktive neue Rubrik erfolgreich nutzen.

Die Herausgeber und der Verlag 